\title{
Comparative analysis on association of fasting and postprandial dyslipidemia with risk of atherosclerosis in early rheumatoid arthritis
}

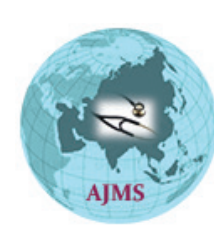

\author{
Puja Kumari Jha', Rafat Sultana Ahmed ${ }^{2}$, Rachna Agarawal ${ }^{3}$ \\ ${ }^{1}$ Senior Resident, Department of Biochemistry, University College of Medical Sciences and Guru TegBahadur Hospital \\ (University of Delhi), Dilshad Garden, Delhi-110095, India, ${ }^{2}$ Director Professor, Department of Biochemistry, University \\ College of Medical Sciences and Guru Teg Bahadur Hospital (University of Delhi), Dilshad Garden, Delhi-110095, \\ India, ${ }^{3}$ Associate Professor, Department of Neurochemistry, Institute of human behavior and allied sciences(IHBAS), \\ Dilshad Garden, Delhi-110095, India
}

Background: Rheumatoid arthritis (RA) patients have higher cardiovascular risk due to dyslipidemia, inflammation etc. The serum lipid levels obtained after 12 hour fasting will not truly reflect atherosclerotic risk associated as non fasting state predominates in routine life. Aim and Objectives: We investigated the frequency of dyslipidemia in early RA cases and healthy controls in both fasting and postprandial state and analyzed the impact of postprandial lipid profile over fasting lipid levels in prediction of atherosclerotic risk. Materials and Methods: This case-control study was conducted at UCMS and GTB Hospital, Delhi. 127 RA cases diagnosed according to revised ACR, 2010 with age and sex matched 127 controls were recruited. Lipid profile parameters were analyzed by commercial kits on auto analyzer. RF, anti-CCP, TNF- $\alpha$ and CRP were measured by ELISA. For statistical analysis unpaired t test and Pearson's correlation was used. Result: The mean disease duration was $7 \pm 2.16$ months. There were significant increased levels of TC, LDL-C, and TAG with reduced serum HDL-C in RA cases. Atherogenic ratio of TC/HDL-C and LDL-C/HDL-C were significantly high in RA. Postprandial lipid analysis revealed significant difference for TAG and HDL-C in RA cases. TAG had a significantly positive correlation with TNF- $\alpha(r=0.234)$ which improved in postprandial state $(r=0.559)$. Significantly negative correlation of HDL-C with ESR $(r=-0.221)$ also improved $(r=-0.421)$ postprandially.Conclusions: Atherogenic dyslipidemia was a significant finding in patients of early RA. Dyslipidemia screening can be recommended in postprandial state.

Key words: Early rheumatoid arthritis; Dyslipidemia; Post prandial; Inflammation; Atherosclerosis

\section{INTRODUCTION}

Rheumatoid arthritis (RA) is an autoimmune disease characterized by chronic and deforming polyarthritis that is accompanied by systemic involvement in many patients. Morning stiffness, fatigue and loss of energy is usually observed in patients with RA. ${ }^{1}$ The prevalence of RA is $0.5 \%$ to $1.0 \%$ worldwide and $0.3 \%$ to $0.9 \%$ in India. ${ }^{2}$

RA causes significant morbidity as a result of synovial inflammation, joint destruction and associated disability. In a landmark study Wolfe et al.(1994) reported a standardized mortality ratio of approximately 2.3 in the largest cohort to date of 3,501 RA patients followed over 35 years, with cardiovascular and cerebrovascular diseases accounting for the excess deaths. ${ }^{3}$ Many other studies have also reported an increase in cardiovascular diseases among RA patients. In active RA, the majority of cardiovascular deaths result from accelerated atherosclerosis. ${ }^{4}$ Traditional risk factor to estimate the atherosclerotic risk are body mass index (BMI), Waist to hip ratio(WHP) and dyslipidemia that includes increased level of TAG, high TC/HDL 
ratio, high LDL/HDL ratio. Other risk factors for atherosclerotic events and cardiovascular disease include male sex, increased age, blood pressure, smoking and diabetes mellitus. ${ }^{5}$ In RA cases there are further increased risk of cardiovascular disease due to presence of many nontraditional risk factors that predicts the disease severity such as inflammatory markers, disease specific markers etc.

Estimation of lipid profile parameters are done mainly in fasting state. According to NCEP (National Cholesterol Education Programme), AACE (American Association of Clinical Endocrinologist), 2017 and European guidelines, the recommended fasting for defined reference value is 12 hour fast. ${ }^{6-8}$ Fasting is defined as no dietary intake for that specified time apart from water and medication. In normal daily life situation this schedule is not followed usually. Therefore the serum lipid levels obtained after 12 hour fasting will not be able to reflect the routine basic lipid levels and thus the risk of atherosclerosis associated. ${ }^{9}$

AACE, 2017 has recommended that HDL-C, non HDL-C and TC can be analyzed in non fasting blood samples and even TAG can be measured in the non-fasting state if fasting determinations are impractical. ${ }^{7}$ The benefits of non fasting lipid analysis over fasting lipids level is quite evident. The whole process of blood collection becomes very simple for everyone starting from patients to laboratories. This may help clinicians also by increasing patient compliance as patients are spared from one extra visit at specific time. ${ }^{10}$

The Canadian Cardiovascular Society's dyslipidemia guidelines 2016, recommends non-fasting lipid and lipoprotein testing which can be performed in adults in whom screening is indicated as part of a comprehensive risk assessment to reduce CVD events. Boccalondro et al. had reported that cases with coronary heart disease used to have a prolonged postprandial lipemia as compared to healthy controls. ${ }^{11}$ Therefore, it is recommended to consider the postprandial period particularly in patients with high risk of cardiovascular disease such as RA to diagnose the cases early.

Lipid metabolism is a very complex process, especially in chronic inflammatory conditions; this may be due to involvement of common proinflammatory cytokines, such as tumor necrosis factor alpha (TNF- $\alpha$ ), interleukins and interferons in the pathogenesis of diseases and lipid metabolism. TNF- $\alpha$ plays a pivotal role in RA synovitis by a variety of mechanism such as by inducing other inflammatory cytokines, stimulating fibroblast, osteoclast and chondrocytes. TNF- $\alpha$, by acting on distant organs like liver, adipose tissue, vascular endothelium and skeletal muscle leads to development of characteristic dyslipidemia, increase in oxidative stress and endothelial dysfunction. ${ }^{12}$

The lipid profile of RA cases is characterized by a decrease in serum levels of HDL-C whereas contrasting results have been reported on the levels of TC and LDL-C. ${ }^{13,14}$ Reduction in HDL-C as a consequence leads to increase in the TC/HDL-C. This ratio is a marker of atherogenicity, which indicates the risk for cardiovascular diseases. ${ }^{14}$ Negative correlation has been reported between disease activity in patients of RA with serum TC and HDL-C levels, indicating a potential role of inflammation for the higher atherosclerotic risk observed in RA. ${ }^{14}$

However, many clinical studies had advised that correlation of lipid level analysis with atherosclerotic risk in non fasting condition is quite equivalent or rather better than fasting in different diseased condition. To the best of our knowledge no study had been conducted to see the difference in lipid level in RA cases in fasting and postprandial state. Therefore, in this study we had attempted to investigate the frequency of dyslipidemia in patients with early RA along with age and sex matched healthy controls in both fasting and postprandial state and analyzed the impact of postprandial lipid profile over fasting lipid levels in prediction of atherosclerotic risk.

\section{MATERIALS AND METHODS}

The present case-control study included 127 cases attending Medicine Outpatient Clinic at University College of Medical Sciences and Guru Teg Bahadur Hospital, Delhi. Diagnosis of RA was made according to guidelines of revised American College of Rheumatology Classification criteria (ACR, 2010). Required sample size was calculated with alpha level as 0.05 and power of the study at $80 \%$. Age and sex matched equal number of healthy controls (HC) were recruited from volunteers and staff of UCMS and GTB Hospital. Prior to participation, the purpose of the study was explained to all the subjects and their written informed consent was taken.

In RA cases there are further increased risk of cardiovascular disease due to presence of many nontraditional risk factors that predicts the disease severity such as inflammatory markers, disease specific markers, physical disability, joint destruction, extra articular manifestations and medications used to treat the disease. To reduce the bias the selected RA case had disease durations of less than one year. They had not received any prior treatment. These early RA cases were just on analgesic and not taking any other disease modifying drugs. Smokers, diabetics, familial dyslipidemic patients, persons with hypothyroidism, liver or kidney 
disease, obesity, cushing's syndrome and those receiving medications affecting lipid metabolism were excluded from the study. Patients with coronary artery disease, chronic obstructive pulmonary disease, emphysema, pregnancy, current malignancy and other chronic inflammatory conditions were also excluded.

We analyzed the association of postprandial lipid profile levels with atherosclerotic risk in early RA cases. Prior to this correlation we tried to find out that whether there is any significant change in fasting and postprandial lipid levels in both RA cases and HC. The objectives of the present study were to analyze the lipid profile parameters in both fasting and postprandial state, correlation of observed lipid levels with serum level of TNF- $\alpha$, C-reactive protein (CRP), erythrocyte sedimentation rate (ESR) and anti-cyclic citrullinated peptide antibody (anti-CCP).

Fasting blood sample was withdrawn from ante-cubital vein under aseptic precautions after 12 hour fast and collected into EDTA and plain vial for complete blood count (CBC), lipid profile, liver function test, erythrocyte sedimentation rate (ESR), fasting glucose, renal profile (urea/creatinine),C-reactive protein (CRP), rheumatoid factor(RF) and anti-cyclic citrullinated peptide antibody (anti-CCP). A second blood sample was drawn next day after 2 hour of having usual breakfast for lipid and glucose analysis.

For every subject, the routine biochemical investigations were performed in the Hospital Laboratory Services, GTB Hospital, Delhi by standard laboratory methods using commercial kits carried on autoanalyzer Unicel DxC 600, Synchron Clinical System(Beckman Coulter Pvt.Ltd., USA). Lipid profile parameters are estimated by enzymatic/colorimetric methods. Both LDL-C and HDL-C was measured directly. CBC performed on Mythic 18 blood cell counter and ESR was measured by modified Westergren method.

Assay for $\operatorname{IgG}$ antibodies to citrullinated protein and RF were done by ELISA (Enzyme Linked Immunosorbent Assay) using commercially available kits (Omega Diagnostics Ltd, Alva, UK). TNF- $\alpha$ was estimated by sandwitch ELISA technique (Diaclone diagnostics, France). CRP level was measured by standard ELISA kit (Ray Biotech, Norcross GA). All standards, patients samples and control samples were run in duplicate.

\section{Ethics}

The study was approved by the Institutional Ethical Committee for human research (IEC-HR). Procedures followed were in accordance with the ethical standards of the responsible committee on human experimentation (institutional) and with the Helsinki Declaration of 1975, as revised in 1983.

\section{Statistics}

All statistical tests were performed using SPSS version 17. For comparisons of demographic profiles, lipid profile parameters and disease biomarkers for RA, unpaired t-test was used. Correlation of dyslipidemia with TNF- $\alpha$, anti-CCP, CRP and ESR were studied with the help of Pearson's correlation. $\mathrm{p}$ value less than $5 \%$ was regarded significant.

\section{RESULTS}

\section{Demographic and disease specific parameters}

The baseline demographic and disease specific markers of the two study groups are presented in Table 1 . The mean disease duration was around seven months. There was a significant difference between RA patients and healthy controls (HC) with respect to Body mass index (BMI). The disease is more prevalent in females as evident from sex distribution in patients. The disease biomarkers and cytokine TNF- $\alpha$ were significantly high in RA patients as compared to HC.

\section{Lipid levels in fasting and postprandial state}

Lipid profile parameters in fasting and postprandial state of both study groups are presented in Table 2. TC, LDL-C, TAG and VLDL-C were significantly higher whereas, HDL-C level was significantly $(\mathrm{p}<0.001)$ lower in RA as compared to HC. LDL-C/HDL-C $(4.2 \pm 0.7)$ and TC/HDL-C(6.7 \pm 0.9$)$ was also significantly high in RA cases than HC. While comparing the fasting lipid profile with postprandial lipid, no significant difference was observed for TC,LDL-C and LDL-C/HDL-C in RA cases.

\begin{tabular}{|c|c|c|}
\hline Parameters & $\begin{array}{c}\text { Healthy } \\
\text { control }(n=127)\end{array}$ & $\begin{array}{l}\text { RA cases } \\
(n=127)\end{array}$ \\
\hline Age (years) & $40.8 \pm 6.7$ & $41.60 \pm 6.3$ \\
\hline Sex ratio (female/male) & $87 / 13$ & $84 / 16$ \\
\hline Duration of RA (months) & NA & $7.0 \pm 2.16$ \\
\hline BMI $\left(\mathrm{kg} / \mathrm{m}^{2}\right)$ & $22.2 \pm 2.0$ & $24.3 \pm 3.7$ * \\
\hline $\mathrm{SBP}(\mathrm{mmHg})$ & $112.0 \pm 6.0$ & $118.0 \pm 6.0$ \\
\hline $\mathrm{DBP}(\mathrm{mmHg})$ & 72.04 .0 & $76.0 \pm 6.0$ \\
\hline $\mathrm{ESR}(\mathrm{mm} / \mathrm{hr})$ & $10.9 \pm 7.1$ & $37.9 \pm 12.82$ * \\
\hline $\mathrm{RF}(\mathrm{U} / \mathrm{ml})$ & $48.2 \pm 25.4$ & $126.6 \pm 23.3^{*}$ \\
\hline a-CCP $(\mathrm{U} / \mathrm{ml})$ & $0.37 \pm 0.6$ & $19.4 \pm 6.34^{*}$ \\
\hline $\mathrm{CRP}(\mathrm{mg} / \mathrm{l})$ & $2.48 \pm 1.73$ & $16.9 \pm 7.3^{*}$ \\
\hline TNF- $\alpha(p g / m l)$ & $35.0 \pm 10.1$ & $14.18 \pm 6.21^{*}$ \\
\hline \multicolumn{3}{|c|}{$\begin{array}{l}\text { Data are expressed as mean } \pm \text { SD. Unpaired 't' test was applied for comparison. } \\
\text { *.Significantly different from healthy control at } p<0.01 \text {. BMI: body mass index; } \\
\text { SBP: Systolic blood pressure; DBP: diastolic blood pressure; ESR: Erythrocyte } \\
\text { sedimentation rate; RF: Rheumatoid factor; a-CCP: Anti cyclic citrullinated peptide; } \\
\text { TNF- } \alpha \text { : Tumor necrosis factor alpha; CRP: C- reactive protein }\end{array}$} \\
\hline
\end{tabular}


Table 2: The lipid profile in fasting and postprandial state of of healthy controls and RA cases

\begin{tabular}{|c|c|c|c|c|}
\hline Parameter & Healthy control (F) & Healthy control (PP) & RA cases (F) & RA cases (PP) \\
\hline $\mathrm{TC}(\mathrm{mg} / \mathrm{dl})$ & $162.4 \pm 21.0$ & $164.3 \pm 22.0$ & $237 \pm 25$ * & $230 \pm 31.3$ \\
\hline LDL-C (mg/dl) & $88.7 \pm 15.8$ & $87.4 \pm 16.0$ & $146.8 \pm 15.3$ * & $140 \pm 33.0$ \\
\hline TAG (mg/dl) & $110.5 \pm 22.2$ & $125 \pm 29.5^{\star *}$ & $182.5 \pm 20.5^{*}$ & $218.7 \pm 41.0^{\# \star *}$ \\
\hline HDL-C (mg/dl) & $49.3 \pm 6.5$ & $48.8 \pm 7.1$ & $35.8 \pm 4.5$ * & $28.4 \pm 3.9^{\#}$ \\
\hline VLDL-C (mg/dl) & $24.1 \pm 7.8$ & $29.0 \pm 5.2$ & $36.5 \pm 4.3$ * & $43.7 \pm 8.2^{\# \star \star}$ \\
\hline $\mathrm{TC} / \mathrm{HDL}-\mathrm{C}$ & $3.35 \pm 0.6$ & $3.36 \pm 0.7$ & $6.7 \pm 0.9^{*}$ & $8.1 \pm 1.1^{\# * *}$ \\
\hline LDL-C/HDL-C & $1.8 \pm 0.4$ & $1.8 \pm 0.6$ & $4.2 \pm 0.7^{*}$ & $4.9 \pm 1.1$ \\
\hline
\end{tabular}

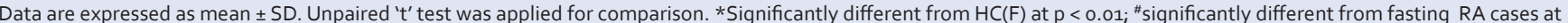
$\mathrm{p}<0.01 ; * *$ significantly different at $\mathrm{p}<0.01$ from HC (PP). HC: healthy control F: Fasting ; PP: Postprandial TC: total cholesterol; HDL-C: high density lipoprotein cholesterol; TAG: triacylglycerol; VLDL-C: very low density lipoprotein cholesterol; LDL-C: low density lipoprotein cholesterol

Significant difference was observed for the parameters TAG, HDL-C and VLDL-C in postprandial state as compared to fasting in RA cases. In $\mathrm{HC}$, apart from TAG which has a significant rise of around 13\% from fasting state, all other parameters have non significant changes.

\section{Atherogenic index (Al)}

TC/HDL-C, LDL-C/HDL-C and TAG/HDL-C are the atherogenic indexes. TC/HDL-C $(8.1 \pm 1.1)$ and TAG/ HDL-C $(0.89 \pm 0.07)$ was significantly higher in postprandial

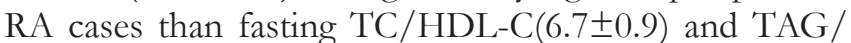
HDL-C $(0.71 \pm 0.08)$

\section{Correlations}

Pearson's correlation was applied to determine the correlation between lipid profile parameters and disease biomarkers in RA cases (Table 3). TAG was found to be positively correlated with $\mathrm{TNF}-\alpha(\mathrm{r}=0.234, \mathrm{p}<0.05)$. In this study, there was positive correlation between serum LDL-C and the serum level of anti-CCP $(r=0.231, \mathrm{p}<0.05)$ where as HDL-C had a significant negative correlation with ESR ( $\mathrm{r}=-0.221, \mathrm{p}<0.05)$. While analyzing the same correlation in postprandial state it was observed that a significant and better positive correlation was found between TAG and TNF -alpha $(\mathrm{r}=0.559, \mathrm{p}<0.05)$. The HDL-C was found to have even more negative correlation with ESR ( $r=-0.421, \mathrm{p}<0.05)$ Although, LDL-C/HDL-C was positively correlated with all the disease biomarkers but not significant. TC/HDL-C was showing a non significant negative correlation with ESR, CRP and anti-CCP.

\section{DISCUSSION}

Cardiovascular disease in RA may result from accelerated atherosclerosis caused by clinical or subclinical vasculitis. ${ }^{15}$ The demographic characteristics and quantitative value of disease specific markers (ESR, RF, anti-CCP, CRP) along with cytokine $\mathrm{TNF}-\alpha$ are presented in Table 1.

According to our results, patients with early RA exhibited significant dyslipidemia. There was a significant increase in serum levels of TC, LDL-C and TAG and a reduction

\begin{tabular}{lcccc}
$\begin{array}{l}\text { Table 3: Correlation between lipid profile } \\
\text { parameters and disease biomarkers in early RA } \\
\text { cases (F) }\end{array}$ & & & & \\
\hline Parameters ( $r$ ) & ESR & CRP & Anti-CCP & TNF- $\alpha$ \\
\hline TAG & 0.144 & 0.03 & 0.103 & $0.234^{*}$ \\
TC & -0.072 & 0.016 & -0.015 & 0.047 \\
LDL-C & 0.052 & 0.023 & $0.231^{*}$ & 0.041 \\
HDL-C & $-0.221^{*}$ & 0.123 & 0.014 & -0.031 \\
TC/HDL-C & -0.102 & -0.016 & -0.034 & 0.023 \\
LDL-C/HDL-C & 0.032 & 0.111 & 0.123 & 0.069
\end{tabular}

Correlation ( $r$ ) value is presented in the table. *Significantly correlated at $p<0.05$ in cases. TC: Total cholesterol; HDL-C: High density lipoprotein cholesterol; TAG: Triacylglycerol; VLDL-C: Very low density lipoprotein cholesterol; LDL-C: Low density lipoprotein cholesterol; ESR: Erythrocyte sedimentation rate CRP: C-reactive protein; a-CCP: Anti cyclic citrullinated peptide; TNF- $\alpha$ :Tumor necrosis factor alpha

in serum HDL-C levels (Table 2). Thus, a significant increase in the ratio of TC/HDL-C or LDL-C/HDL-C is observed in early RA patients. The altered lipid profile of early rheumatoid patients in this study is highlighting its atherogenic phenotype. All three elements of atherogenic dyslipidemia (elevated levels of TAG and small-dense LDL and low levels of HDL-C) per se have been recognized as independent risk factor for cardiovascular disease The lipid profile of patients with RA has been evaluated in several studies. Some of these studies have reported lower levels of HDL-C and TC, higher serum concentrations of lipoprotein (a) and higher TC/HDL-C and LDL-C/ HDL-C ratios in active and/or untreated disease than in the general population. ${ }^{16,17}$ These variations can be due to effect of different drug of RA on lipid profile. Effect of medication is nullified in this study as we had selected the early RA cases.

While comparing the fasting lipid profile with postprandial lipid, significant difference was observed for TAG, HDL-C, VLDL-C and TC/HDL-C in RA cases (Table 2).Several studies have reported that non-fasting TAG levels are a better risk predictor for ASCVD than the fasting TAG levels. ${ }^{18}$ In the prospective study reported by Cullen et al. high TAG levels was supposed to be independent risk for cardiovascular events after balancing the other risk factors like LDL-C,HDL-C etc. ${ }^{19}$ We observed a non significant 
decline in TC and LDL-C in postprandial state than fasting. This decrease may be due to the hemodilution. ${ }^{20}$ The increase in TAG can be explained by the dietary fat content; the significant decline in HDL-C is due to exchange of cholesterol ester and TAG between TAG rich lipoproteins and HDL-C. ${ }^{21}$ Higher TAG levels lead to increased formation of small dense LDL which has a low clearance from systemic circulation due to reduced affinity to LDL. ${ }^{22}$ Increased VLDL-C produce small HDL by action of hepatic lipase and cholesterol ester transfer protein. This small HDL has a high clearance from the circulation leading to decrease in the plasma level of HDL-C and apolipoprotein A-I. Hence, increased production of VLDL and TAG ultimately leads to atherogenic reduction of HDL. ${ }^{23}$ Again low level of HDL-C indicates decreased reverse cholesterol transport and further increase in TAG rich lipoprotein and more risk of atherosclerosis in cases. Thus the triple marker of high TAG and VLDL with low HDL are better predictor of atherosclerosis. In this study, we had found a significant high TC/HDL-C and LDL-C/HDL-C in RA cases than HC (Table 2). TC/HDL-C was significantly higher in RA cases in postprandialstate. This ratio represents an atherogenic index, which is an important prognostic marker for cardiovascular disease. The risk of myocardial infarction increases considerably when TC/HDL-C ratio is higher than five and it should ideally be four or less. ${ }^{5,13} \mathrm{In}$ healthy controls there was significant increase in TAG only. Therefore with appropriate reference value for TAG, lipid profile analysis can be recommended in postprandial state.

Analysis of correlation nontraditional risk factor with traditional risk factor of atherosclerosis i.e. inflammatory and disease specific marker with TAG, TC/HDL-c and LDL-c/HDL-c. We have reported a significant and positive correlation TNF- $\alpha$ with TAG (Tables 3 and 4).TNF- $\alpha$ increases free fatty acid by stimulating lipolysis in adipose tissue and thus increases TAG synthesis in liver and at vascular endothelium reduces lipoprotein lipase activity. Thus chronic elevation in TNF- $\alpha$ is able to generate a spectrum of pro-atherogenic changes. ${ }^{12}$ While analyzing the same correlation in postprandial state it was observed that a significant and better positive correlation was found between TAG and TNF -alpha $(r=0.559, \mathrm{p}<0.05)$. A significant and negative correlation was observed between HDL-C and ESR (Tables 3 and 4). ESR being acute phase reactant indicates the disease activity. This correlation can be explained anti-oxidant and other protective properties of HDL-C. ${ }^{23}$ Anti-atherogenic activities of HDL-C are attributed to reverse cholesterol transport and host of other protective activities such as counteracting LDL oxidation, anti-inflammatory, antithrombotic/profibrinolytic and vasoprotective (facilitating vascular relaxation and inhibiting leukocyte chemotaxis and adhesion) effects. ${ }^{23}$

\begin{tabular}{|c|c|c|c|c|}
\hline Parameters ( $r$ ) & ESR & CRP & Anti-CCP & TNF- $\alpha$ \\
\hline TAG & 0.161 & 0.11 & 0.112 & $0.559^{*}$ \\
\hline $\mathrm{TC}$ & -0.06 & 0.021 & -0.011 & 0.041 \\
\hline LDL-C & 0.047 & 0.018 & 0.121 & 0.031 \\
\hline HDL-C & $-0.421^{*}$ & 0.104 & 0.021 & -0.041 \\
\hline TC/HDL-C & -0.092 & -0.024 & -0.053 & 0.083 \\
\hline LDL-C/HDL-C & 0.052 & 0.113 & 0.134 & 0.101 \\
\hline
\end{tabular}

Correlation $(r)$ value is presented in the table. *Significantly correlated at $p<0.05$ in cases. TC: Total cholesterol; HDL-C: High density lipoprotein cholesterol; TAG: Triacylglycerol; VLDL-C: Very low density lipoprotein cholesterol; LDL-C: Low density lipoprotein cholesterol; ESR: erythrocyte sedimentation rate CRP: C- reactive protein; a-CCP: Anti cyclic citrullinated peptide; TNF- $\alpha$ : Tumor necrosis factor alpha

The higher level of disease specific inflammatory markers along with high inflammatory cytokine (TNF- $\alpha$ ) level induces lipid alteration in RA cases and there correlation is more significant in postprandial state.

\section{CONCLUSION}

Patients with early RA exhibited an atherogenic lipid profile. Postprandial lipid analysis show significant rise in serum levels of TAG, HDL-C, VLDL-C and TC/HDL-C with a significant reduction in serum HDL-C levels in RA cases than fasting state. We observed a a significantly positive correlation of TNF- $\alpha$ with TAG and significantly negative correlation of HDL-C with ESR in both fasting and postprandial state. As primary screening for dyslipidemia and its risk with atherosclerosis in early RA is mandatory, these findings favor the importance of recommending non fasting lipid profile analysis for screening of risk the atherosclerotic risk in RA cases. The analytical methodology used for lipid analysis had a good performance in both fasting and postprandial state.

\section{Study limitations}

This study basically focused on the analytic issue of lipid parameters in laboratories in fasting and postprandial state. There are many more modulators of lipid parameters in RA cases such as physical activity, obesity etc., those have not been considered. The meal was not standardized. Samples can be analyzed at different time intervals. The fact that this study was conducted at a single center may have introduced bias. To investigate the variation in DAS28, CRP, and lipids over time would also be interesting.

\section{ACKNOWLEDGEMENT}

One of the authors Dr. Puja Kumari Jha is thankful to the Indian Council of Medical Research, New Delhi for providing partial funding for this study (MD Thesis Grant). 


\section{REFERENCES}

1. Bergsten U, Bergman S, Fridlund B and Arvidsson B. Striving for a good life - the management of rheumatoid arthritis as experienced by patients. The open nursing journal 2011; 5:95.

2. Malaviya A, Kapoor S, Singh R, Kumar A and Pande I. Prevalence of rheumatoid arthritis in the adult Indian population. Rheumatology International 1993;13(4):131-134.

3. Wolfe F, Mitchell DM, Sibley JT, Fries JF, Bloch DA, Williams CA, et al. The mortality of rheumatoid arthritis. Arthritis \& Rheumatology 1994; 37(4):481-494.

4. Van Doornum S, McColl G and Wicks I. Accelerated atherosclerosis: an extraarticular feature of rheumatoid arthritis? Arthritis \& Rheumatology 2002;46(4):862-873.

5. Cui Y, Blumenthal RS, Flaws JA, Whiteman MK, Langenberg $P$, Bachorik PS, et al. Non-high-density lipoprotein cholesterol level as a predictor of cardiovascular disease mortality. Archives of Internal Medicine 2001;161(11):1413-1419.

6. Third report of the National Cholesterol Education Program (NCEP) Expert panel on detection, evaluation and treatment of high blood cholesterol in adults (adult treatment panel III) final report. Circulation 2002;106:3143-3421.

7. American association of clinical endocrinologist and American College Of Endocrinology Guidelines For Management of Dyslipidemia and Prevention of Cardiovascular Diseases. Endocrine Practice 2017;23(Suppl 2): 1-87.

8. De Backer G, Ambrosioni E, Borch-Johnsen K, Brotons C, Cifkova R, Dallongeville $\mathrm{J}$, et al. European guidelines on cardiovascular disease prevention in clinical practice: third joint task force of European and other societies on cardiovascular disease prevention in clinical practice (constituted by representatives of eight societies and by invited experts). European heart journal 2003; 24(17):1601-1610.

9. Simundic AM, Cornes M, Grankvist K, Lippi G and Nybo M. Standardization of collection requirements for fasting samples: for the Working Group on Preanalytical Phase (WG-PA) of the European Federation of Clinical Chemistry and Laboratory Medicine (EFLM). International Journal of Clinical Chemistry 2014; 432:33-37.

10. Khera AV and Mora S. Fasting for lipid testing: is it worth the trouble? Archives of Internal Medicine 2012;172:1710-1712.

11. Boccalandro F, Farias J, Boccalandro $C$ and Vaisman D. Frequency of postprandial lipemia after a first acute coronary event (unstable angina pectoris or non-ST-segment elevation acute myocardial infarction) and the effects of atenolol on the lipemia. The American Journal of Cardiology 2002; 90(2):153-156.

12. Sattar N, McCarey DW, Capell $\mathrm{H}$ and Mclnnes IB. Explaining how "high-grade" systemic inflammation accelerates vascular risk in rheumatoid arthritis. Circulation 2003;108(24):2957-2963.

13. Boers M, Nurmohamed M, Doelman C, Lard L, Verhoeven A, Voskuyl A, et al. Influence of glucocorticoids and disease activity on total and high density lipoprotein cholesterol in patients with rheumatoid arthritis. Annals of The Rheumatic Diseases 2003;62(9):842-845.

14. Park YB, Lee S, Lee W, Suh C, Lee C, Lee C, et al. Lipid profiles in untreated patients with rheumatoid arthritis. The Journal of Rheumatology 1999;26(8):1701-1704.

15. Bacons $P$ and Kitas $G$. The significance of vascular inflammation in rheumatoid arthritis. Annals of the rheumatic diseases 1994;53(10):621.

16. Situnayake RD and Kitas G. Dyslipidemia and rheumatoid arthritis. Annals of the rheumatic diseases.1997;56(6):341-2. Epub 1997/06/01.

17. Del Rincón I, Williams K, Stern MP, Freeman GL and Escalante A. High incidence of cardiovascular events in a rheumatoid arthritis cohort not explained by traditional cardiac risk factors. Arthritis \& Rheumatology 2001;44(12):2737-2745.

18. Nordestgaard BG, Benn M, Schnohr P and Tybjaerg-Hansen A. Nonfasting triglycerides and risk of myocardial infarction, ischemic heart disease and death in men and women. JAMA 2007; 298:299-308.

19. Cullen P. Evidence that triglycerides are independent coronary heart disease risk factors. The American Journal of Cardiology 2000; 86: 943-949.

20. Wilder LB, Bachorik PS, Finney CA, Moy TF and Becker DM. The effect of fasting status on the determination of low-density and high-density lipoprotein cholesterol. The American Journal of Medicine 1995;99:374-377.

21. Zilversmit DB. Atherogenic nature of triglycerides, postprandial lipidemia, and triglyceride-rich remnant lipoproteins. Clinical chemistry 1995; 41:153-158.

22. Musunuru K. Atherogenic dyslipidemia: cardiovascular risk and dietary intervention. Lipids. 2010;45(10):907-914.

23. Kontush A, Chantepie S and Chapman MJ. Small, dense HDL particles exert potent protection of atherogenic LDL against oxidative stress. Arteriosclerosis, Thrombosis, and Vascular Biology 2003;23(10):1881-1888.

\footnotetext{
Authors Contribution:

PKJ-Concept and design of the study, reviewed the literature, collection of data, statistically analyzed and interpreted, manuscript preparation and revision of the manuscript; RSA- Concept and critical revision of the manuscript; RA- Review of study and manuscript editing.

Work attributed to:

Department of Biochemistry, University College of Medical Sciences and Guru Teg Bahadur Hospital (University of Delhi), Dilshad Garden, Delhi-110095, India

Orcid ID:

Dr. Puja Kumari Jha - iD https://orcid.org/0000-0002-4662-9897

Dr. Rafat Sultana Ahmed - iD https://orcid.org/0000-0003-3421-0236

Source of Support: There was no funding source for execution of this study apart from institutional support, Conflict of Interest: The authors declare that there is no conflict of interest.
} 